\title{
Serum Aminotransferases in Thai Children With Dengue Infection
}

\author{
Olarn Prommalikit, ${ }^{1, *}$ Usa Thisyakorn, ${ }^{2}$ and Chule Thisyakorn ${ }^{2}$ \\ ${ }^{1}$ Department of Pediatrics, Faculty of Medicine, Srinakharinwirot University, Nakhon-Nayok, Thailand \\ ${ }^{2}$ Department of Pediatrics, Faculty of Medicine, Chulalongkorn University, Bangkok, Thailand \\ *Corresponding author: Olarn Prommalikit, Department of Pediatrics, Faculty of Medicine, Srinakharinwirot University, P. O. Box: 26120, Nakhon-Nayok, Thailand. Tel:+66-37395085(10920), \\ Fax:+66-37395087, E-mail: drolarnp2002@yahoo.com
}

Received 2015 January 22; Accepted 2015 September 26

\begin{abstract}
Background: Hepatic manifestations are one of the unusual manifestations of dengue infection.

Objectives: We conducted this study in order to study the pattern of serum aminotransferases and sequential changes before and after shock in Thai children with dengue infection.

Patients and Methods: Children who were clinically and serologically diagnosed as dengue infection and were admitted to King Chulalongkorn Memorial Hospital during a peroid of one year were enrolled. They were clinically classified into a non-shock group and a shock group. The majority of serum aminotransferases including aspartate aminotransferase (AST) and alanine aminotransferase (ALT) were obtained within a week after the onset of fever and until 3 days after shock in the shock group. Student t-test and median in boxplot form were used for statistical analysis.

Results: We enrolled 127 children with a mean age of $7.6 \pm 3.6$ years. The incidence of abnormal AST and ALT levels was $97.4 \%$ and $50.0 \%$ in the shock group, and $91.8 \%$ and $44.9 \%$ in the non-shock group respectively. $29 \%$ and $15.4 \%$ of the patients in shock group and only $10.2 \%$ and 4.1\% in non-shock group had the respective AST and ALT levels > $200 \mathrm{U} / \mathrm{L}$. Serum aminotransferase levels were significantly higher in the shock group when compared to the non-shock group. AST tended to increase starting from one day before shock and continued to increase within a few days whereas ALT was less likely to be affected.

Conclusions: Elevated serum aminotransferases are a common finding in children with dengue infection and the levels of AST are higher than those of ALT. Patients with shock have significantly higher aminotransferase levels that increase up to 3 days after shock.
\end{abstract}

Keywords: Dengue, Serum Aminotransferases, Infection

\section{Background}

Dengue virus infections cause a spectrum of illness ranging from asymptomatic, mild undifferentiated fever, dengue fever (DF), dengue hemorrhagic fever (DHF) and dengue shock syndrome (DSS) (1). There have been increasing reports of dengue infection with unusual manifestations (2). Hepatic manifestations are one of unusual manifestations including the elevation of serum aminotransferases, fulminant hepatic failure, hepatic encephalopathy, severe coagulopathy and Reye-like syndrome (3). However, there is a report concerning sequential changes of serum aminotransferases in patients with DF and none for DSS (4).

\section{Objectives}

Therefore, we conducted this study in order to study the pattern of serum aminotransferases and sequential changes before and after shock in Thai children with dengue infection.

\section{Patients and Methods}

This study was conducted during a calendar year in the Department of Pediatrics at King Chulalongkorn Memorial Hospital, a tertiary care hospital in Bangkok, Thailand. Inclusion criteria included: 1) children aged 0 - 15 years who were admitted to King Chulalongkorn Memorial Hospital, 2) clinical diagnosis of dengue virus infection, using World Health Organization (WHO) criteria (5, 6), and 3) confirmed dengue serological diagnosis, using hemagglutination inhibition (HI) test and enzymelinked immunosorbent assay (ELISA) from The Armed Forces Research Institute of Medical Sciences (AFRIMS). The patients were clinically classified into a non-shock group (DF, DHF grade I \& II) and a shock group (DHF grade III \& IV) according to WHO criteria $(5,6)$. Data collected from medical records included age and sex of the patients, clinical presentation and grading of the disease. The majority of serum aminotransferases including aspartate aminotransferase (AST) and alanine aminotransferase (ALT) were obtained within a week after the onset of fever and until 3 days after shock in the shock group. Abnormal AST and ALT levels were $>38$ U/L. For sequential changes before and after shock, we defined the day 0 was

Copyright (C) 2015, Growth \& Development Research Center. This is an open-access article distributed under the terms of the Creative Commons Attribution-NonCommercial 4.0 International License (http://creativecommons.org/licenses/by-nc/4.0/) which permits copy and redistribute the material just in noncommercial usages, provided the original work is properly cited. 
the day of shock. The day prior to shock was designated day-1 Days after shock were day 1, day 2, and so forth. Serum aminotransferases in each day were studied. Descriptive data were analyzed using mean and percentage. Student's t-test and median in boxplot form were used for statistical analysis. P value $<0.05$ was considered to be significant.

\section{Results}

We enrolled 127 children who were clinically and serologically diagnosed as dengue virus infected. Of these, 59 were males and 68 females. Age distribution varied from 1 year to 15 years with a mean age of $7.6 \pm 3.6$ years. Concerning disease severity, 49 were classified as a non-shock group i.e. DF (19), DHF grade I (8) and DHF grade II (22), whereas 78 were classified as a shock group i.e. DHF grade III (68) and DHF grade IV (10). The incidence of abnormal AST and ALT levels (AST and ALT > $38 \mathrm{U} / \mathrm{L}$ ) were $97.4 \%$ and $50.0 \%$ in the shock group, and $91.8 \%$ and $44.9 \%$ in the nonshock group respectively. There was no significant difference in serum aminotransferases at cutoff level of $>$ $38 \mathrm{U} / \mathrm{L}$ between groups. Twenty-nine percent and $15.4 \%$ of the patients in the shock group and only $10.2 \%$ and $4.1 \%$ in the non-shock group had the respective AST and ALT levels $>200 \mathrm{U} / \mathrm{L}$. There was significant difference in serum aminotransferases at cutoff level of $>200 \mathrm{U} / \mathrm{L}$ between groups $(\mathrm{P}<0.05)$. Abnormal AST and ALT levels classified by severity of dengue infection are shown in Tables 1 and 2. Serum aminotransferase levels were significantly higher in the shock group when compared to the non-shock group. Concerning sequential changes of aminotransferases, AST tended to increase starting from one day before shock and was continuously increasing up to 3 days after shock whereas ALT was less likely to be affected (Figure 1).

Table 1. Percentage of Patients With Abnormal AST and ALT Classified by Severity of Dengue Infection ${ }^{\mathrm{a}}$

\begin{tabular}{lccc}
\hline Parameter, U/L & $\begin{array}{c}\text { Non-Shock } \\
(\mathbf{n}=\mathbf{4 9})^{\mathbf{b}}\end{array}$ & Shock $(\mathbf{n}=\mathbf{7 8})^{\mathbf{b}}$ & PValue \\
\hline AST $>\mathbf{3 8}$ & 91.8 & 97.4 & NS \\
\hline AST $>\mathbf{2 0 0}$ & 10.2 & 29.5 & $<0.05$ \\
ALT $>\mathbf{3 8}$ & 44.9 & 50.0 & NS \\
ALT $>\mathbf{2 0 0}$ & 4.1 & 15.4 & $<0.05$ \\
\hline
\end{tabular}

abbreviations: ALT, alanine aminotransferase; AST, aspartate aminotransferase.

balues unit is \%.

Table 2. The Difference of Serum Aminotransferase Levels Classified by Severity of Dengue Infection ${ }^{\mathrm{a}}$

\begin{tabular}{lccc}
\hline $\begin{array}{l}\text { Parameter, } \\
\text { U/L }\end{array}$ & Non-shock $^{\mathbf{b}}$ & Shock $^{\mathbf{b}}$ & P Value \\
\hline AST & $143.4 \pm 240.5$ & $474.2 \pm 1167.3$ & $<0.05$ \\
ALT & $49.4 \pm 58.3$ & $242.4 \pm 714.2$ & $<0.05$ \\
\hline $\begin{array}{l}\text { a Abbreviations: } \\
\text { aminotransferase. }\end{array}$ & ALT, alanine & aminotransferase; & AST, aspartate \\
balues are presented as mean \pm SD. & &
\end{tabular}

Figure 1. Sequential Changes of Serum Aminotransferases Before and Af ter Shock

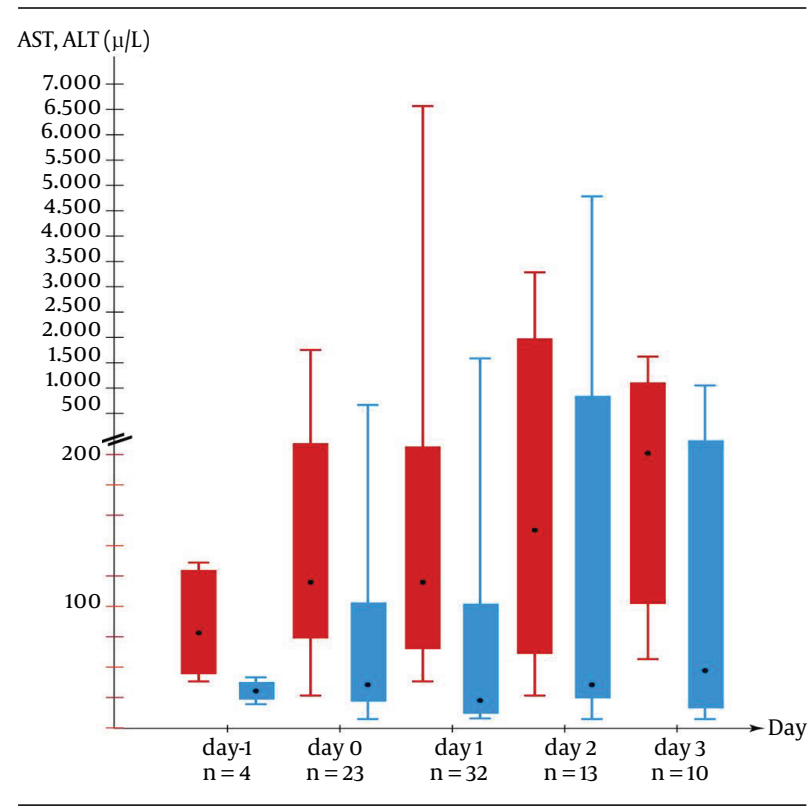

Red bar $=$ AST, blue bar $=$ ALT .

\section{Discussion}

Hepatic dysfunction is common in dengue infection and degree of dysfunction varies from mild injury with elevation of serum aminotransferases to fulminant hepatic failure. Dengue infection is the most important cause of acute hepatic failure in children aged 1 - 15 years contributing to $34.3 \%$ of the cases (7). Serum aminotransferase levels are useful in predicting the occurrence of hepatic dysfunction and spontaneous bleeding (8). In our study, hepatic dysfunction was found in both the non-shock group (4.1\%) and the shock group (15.4\%) as defined by those who had ALT more than 5 times upper normal values (ALT > 200 $\mathrm{U} / \mathrm{L}$ ). Similar results were obtained by Jagadishkumar et al. (9), who showed that hepatic dysfunction was found in DF, DHF and DSS patients and the ALT levels were significantly more in DSS and DHF group. The rise of AST levels were higher than the rise of ALT levels in both the nonshock group and the shock group and this was distinct from other viral hepatitis (10). Mahmuduzzaman et al. reported the increase in serum aminotransferases, mainly AST which has been associated with the disease severity and served as an early indicator of dengue infection (11). For patients with DSS in this study, AST tended to increase starting from one day before shock and continued to increase within a few days whereas ALT was less likely to be affected. Although the study from Brazil in 2012 reported that increases in the AST enzyme occurred at the beginning of the disease and remained stable for all clinical forms (classical dengue fever, DHF and severe dengue); this was more pronounced in the under 15-year-old age group. While ALT started with above normal values in the 
severe form and remained steady throughout the course of the disease; in the classic and hemorrhagic forms, the increases occurred progressively (12). The liver is another target site of dengue virus which was demonstrated by confirming dengue-2 virus replication in human liver cell lines and liver cells of mice $(13,14)$. Patients with DSS have significantly higher aminotransferases. Apart from dengue virus, the heptocellular damage in this condition is probably caused by hypoxemia as a result of hypovolemic shock or host's response to infection (15). In conclusion, elevated serum aminotransferases are a common finding in children with dengue infection. In dengue infection, the level of AST is higher than ALT and serum aminotransferase levels are useful in the differential diagnosis of dengue infection from other viral hepatitis. Patients with shock have significantly higher aminotransferases and the levels are still continuously increasing up to 3 days after shock.

\section{References}

1. Prommalikit O, Pancharoen C, Nisalak A, Thisyakorn U, Thisyakorn C. Pathogenesis of dengue shock syndrome: immune enhancement or viral virulence. Asian Oceanian J Paediatr Child Health. 2004;3:26-9.

2. Hemungkorn M, Thisyakorn U, Thisyakorn C. Dengue infection: a growing global health threat. Biosci Trends. 2007;1(2):90-6. [PubMed: 20103874]

3. Lum LC, Lam SK, George R, Devi S. Fulminant hepatitis in dengue infection. Southeast Asian J Trop Med Public Health. 1993;24(3):46771. [PubMed: 8160054]

4. Wang LY, Chang WY, Lu SN, Chen TP. Sequential changes of serum transaminase and abdominal sonography in patients with sus- pected dengue fever. Gaoxiong Yi Xue Ke Xue Za Zhi. 1990;6(9):4839. [PubMed: 2213970]

5. World Health Organization .In: Dengue haemorrhagic fever: diagnosis, treatment and control. 2nd, editor. Geneva : World Health Organization; 1997.

6. World Health Organization. Comprehensive guidelines for prevention and control of dengue and dengue haemorrhagic fever. 2011.

7. Poovorawan Y, Hutagalung Y, Chongsrisawat V, Boudville I, Bock HL. Dengue virus infection: a major cause of acute hepatic failure in Thai children. Ann Trop Paediatr. 2006;26(1):17-23. doi: 10.1179/146532806X90565. [PubMed:16494700]

8. Petdachai W. Hepatic Dysfunction in Children with Dengue Shock Syndrome. Dengue Bulletin. 2005; 29:112-8.

9. Jagadishkumar K, Jain P, Manjunath VG, Umesh L. Hepatic involvement in dengue Fever in children. Iran J Pediatr. 2012;22(2):231-6. [PubMed: 23056891]

10. Nimmannitya S, Thisyakorn U, Hemsrichart V. Dengue haemorrhagic fever with unusual manifestations. Southeast Asian J Trop Med Public Health. 1987;18(3):398-406. [PubMed: 3433170]

11. Mahmuduzzaman M, Chowdhury AS, Ghosh DK, Kabir IM, Rahman MA, Ali MS. Serum transaminase level changes in dengue fever and its correlation with disease severity. Mymensingh Med J. 2011;20(3):349-55. [PubMed: 21804492]

12. Azin FR, Goncalves RP, Pitombeira MH, Lima DM, Branco IC. Dengue: profile of hematological and biochemical dynamics. Rev Bras Hematol Hemoter. 2012;34(1):36-41. doi: 10.5581/15168484.20120012. [PubMed: 23049382]

13. Lin YL, Liu CC, Lei HY, Yeh TM, Lin YS, Chen RM, et al. Infection of five human liver cell lines by dengue-2 virus. $J$ Med Virol. 2000;60(4):425-31. [PubMed:10686026]

14. Paes MV, Lenzi HL, Nogueira AC, Nuovo GJ, Pinhao AT, Mota EM, et al. Hepatic damage associated with dengue-2 virus replication in liver cells of BALB/c mice. Lab Invest. 2009;89(10):1140-51. doi: 10.1038/labinvest.2009.83. [PubMed:19721415]

15. Pancharoen C, Rungsarannont A, Thisyakorn U. Hepatic dysfunction in dengue patients with various severity. J Med Assoc Thai. 2002;85 Suppl 1:S298-301. [PubMed: 12188427] 